\title{
Projeto Mutirão de Catarata em centro de referência oftalmológico, em Pernambuco: perfil, grrau de satisfação e benefício visual do usuário
}

\author{
Medical Social Cataract Project ("Mutirão de Catarata") in an ophthalmologic center- \\ Pernambuco State, Brazil: user profile, patient satisfaction and visual outcome
}

\author{
Liana 0 Ventura ${ }^{1}$ \\ Carlos Teixeira Brandt ${ }^{2}$
}

Doutora em Oftalmologia pela Universidade Federal de Minas Gerais. Coordenadora do Curso de Residência Médica e Especialização em Oftalmologia da Fundação Altino Ventura. Aluna do Curso de Pós-Graduação Mestrado Profissionalizante em Administração da Prática Oftalmológica pela Universidade Federal de São Paulo - UNIFESP.

2 Professor Titular da Disciplina de Cirurgia Pediátrica da Universidade Federal de Pernambuco. Coordenador do Departamento de Investigação Científica da Fundação Altino Ventura.

Endereço para correspondência: Fundação Altino Ventura, Rua da Soledade, 170 - Recife (PE) CEP 50070-040

E-mail: fav@fundacaoaltinoventura.org.br

Recebido para publicação em 29.07.2003

Versão revisada recebida em 29.10.2003

Aprovação em 14.11.2003

Nota Editorial: Pela análise deste trabalho e por sua anuência na divulgação desta nota, agradecemos ao Dr. José Beniz Neto.

\begin{tabular}{|c|}
\hline RESUMO \\
\hline 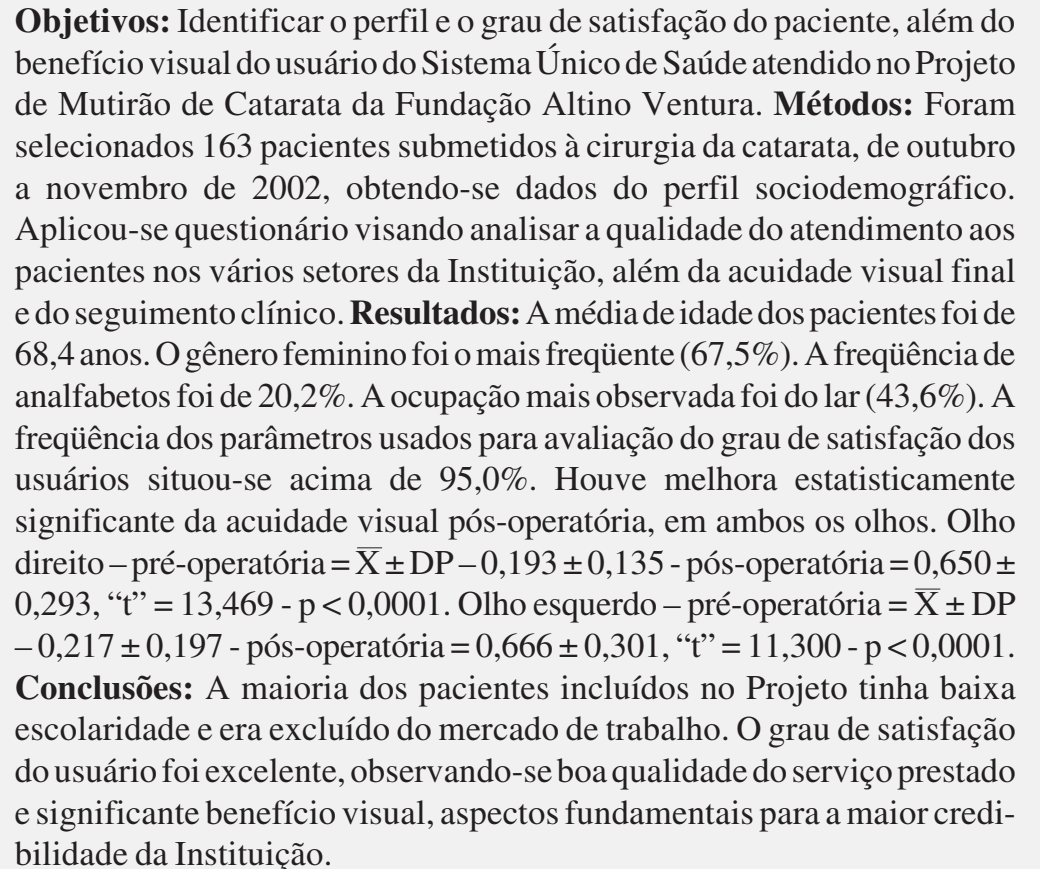 \\
\hline
\end{tabular}

Descritores: Catarata/patologia; Cegueira; Satisfação do paciente; Qualidade de vida; Serviços de saúde comunitária

\section{INTRODUÇ̃̃̃O}

A catarata é a principal causa de cegueira curável, representando um problema de saúde pública que interfere negativamente na qualidade de vida dos pacientes ${ }^{(1-3)}$. Além da escassez de recursos para atender as carências no setor ${ }^{(4)}$, as cirurgias são limitadas pela dificuldade que o idoso enfrenta para chegar ao tratamento ${ }^{(2,5-6)}$.

Nos Estados Unidos são realizados, anualmente, dois milhões de cirurgias de catarata ${ }^{(7)}$; mas, na Índia essa cirurgia é realizada em pequeno percentual dos $\operatorname{casos}^{(3)}$. No Brasil, no início da década de 90, havia cerca de 350 mil cegos por catarata ${ }^{(2,4)}$, realizando-se $20 \%$ das cirurgias necessárias $^{(2)}$. Uma dificuldade era o acesso ao serviço especializado, gerando uma demanda reprimida. Dessa forma, programas de prevenção e controle foram estabelecidos para diminuir esse déficit ${ }^{(2,4-6,8-9)}$. Nesse sentido, em 2000, 
foram realizados 250 mil cirurgias $^{(2)}$, permitindo que os pacientes fossem re-incluídos socialmente ${ }^{(10,11)}$.

Sendo os recursos limitados, os aspectos qualitativos dos projetos de prevenção da cegueira e reabilitação visual não podem ser ignorados ${ }^{(4,11-13)}$.

A presente investigação foi motivada pelo fato de que, em estudos anteriores foram analisados aspectos quantitativos e qualitativos dos Projetos de Catarata no Sul do Brasil, mas, não existiam dados disponíveis no Nordeste ${ }^{(4-6,8-9)}$. Objetivouse identificar o perfil e o grau de satisfação do paciente, além do benefício visual do usuário do Sistema Único de Saúde atendido no Projeto de Mutirão de Catarata da Fundação Altino Ventura.

\section{MÉTODOS}

Foram selecionados os pacientes no pós-operatório de rotina, nos meses de outubro e novembro de 2002, na Fundação Altino Ventura (FAV) - Pernambuco, Brasil.

Foram incluídos 163 voluntários com mais de 40 anos, submetidos à cirurgia de catarata. Os pacientes foram avaliados através de questionário fechado, coleta de dados do prontuário médico e exame oftalmológico completo. O questionário dirigido de múltipla escolha foi aplicado por profissional treinado, não envolvido na análise dos dados.

Foram analisados: idade, gênero, procedência, escolaridade, ocupação atual, grau de satisfação do paciente com a instituição - se repetiria a cirurgia na Instituição e se indicaria a terapia no serviço para amigos ou familiares, opinião do paciente quanto à validade do procedimento cirúrgico, forma de referência dos pacientes, agendamento da consulta, grau de dificuldade na localização da instituição, qualidade e pontualidade do atendimento nos diversos setores do serviço e queixas de complicações.

Identificou-se a acuidade visual pré e pós-operatória e a técnica cirúrgica utilizada.

Foram analisados: acuidade visual corrigida final, complicações pós-operatórias tardias relacionadas à cirurgia da catarata e o tempo de seguimento do paciente. A aferição da acuidade visual foi realizada com a melhor correção óptica, utilizando-se a tabela de Snellen, expressa de acordo com a classificação ICD-9-CM (WHO/ICO) ${ }^{(14)}$ : I - Visão normal ou próxima do normal - acuidade visual entre 2,0 e 0,3; II - Baixa visão moderada - acuidade visual entre 0,25 e 0,12 ; III - Baixa visão grave - acuidade visual entre 0,10 e 0,05; e IV - Baixa visão profunda - acuidade visual inferior a 0,05 . Não foram incluídas as acuidades visuais referentes à visão próxima a cegueira e a cegueira total. Em 81 olhos direitos e 57 esquerdos esse parâmetro foi avaliado como variável contínua.

O Projeto foi aprovado pela Comissão de Ética em Pesquisa da FAV e da UNIFESP, tendo o paciente assinado o termo de consentimento livre e esclarecido.

Os resultados das variáveis contínuas foram expressas por suas médias e respectivos desvios padrão ou erros padrão da média. Os resultados das variáveis categóricas foram expressos por suas freqüências. Foi utilizado o teste "t" de Student para amostras pareadas e não-pareadas no sentido da verificação de possível diferença entre médias. Foi utilizado o teste do quiquadrado para verificação de possíveis diferenças entre frequiências. Aceitou-se $\mathrm{p}<0,05$ para rejeição de hipótese de nulidade.

\section{RESULTADOS}

A idade variou de 40 a 97 anos, média de $68 \pm 10$ anos. A maior freqüência ocorreu entre 60 a 79 anos (74\%). Houve maior freqüência de mulheres (67\%). A maioria dos pacientes (80\%), era proveniente da zona metropolitana do Recife. Os outros procediam: $6 \%$ da zona da mata; $5 \%$ do agreste; $6 \%$ do sertão; e 3\% de outros estados.

Quanto à escolaridade, 20\% eram analfabetos, $67 \%$ cursavam o ensino fundamental, $12 \%$ o ensino médio e $1 \%$ tinha o curso superior completo.

Quanto à ocupação, $14 \%$ estavam empregados, enquanto $12 \%$ desempregados. Quanto à atividade $44 \%$ tinham atividades do lar, 26\% eram aposentados e 4\% autônomos.

A avaliação do grau de satisfação com a Instituição e validade da cirurgia está na tabela 1.

A maioria dos usuários não teve dificuldade em encontrar a Instituição tabela 2.

Na tabela 3 encontram-se os resultados da avaliação da qualidade dos atendimentos nos diversos setores da Instituição.

A distribuição das freqüências da pontualidade nos diversos setores do atendimento oftalmológico na Instituição está na tabela 4 .

\begin{tabular}{|c|c|c|c|c|}
\hline \multirow{2}{*}{$\begin{array}{l}\text { Parâmetros de avaliação da qualidade } \\
\text { do atendimento na Instituição }\end{array}$} & \multicolumn{2}{|c|}{ Sim } & \multicolumn{2}{|c|}{ Não } \\
\hline & $\bar{n}$ & $\overline{\%}$ & $\mathbf{n}$ & $\%$ \\
\hline Opinião quanto à repetição da cirurgia & 154 & 94 & 9 & 5 \\
\hline Recomendação da Instituição & 159 & 97 & 4 & 2 \\
\hline Opinião quanto à validade da cirurgia & 159 & 97 & 5 & 3 \\
\hline
\end{tabular}

\begin{tabular}{|lcr|}
\hline \multicolumn{3}{|c|}{ Tabela 2. Freqüências das dificuldades em encontrar a Instituição } \\
Dificuldades & $\mathbf{n}$ & $\%$ \\
Sim & 44 & 27 \\
Regular & 7 & 4 \\
Não & 112 & 69 \\
\hline
\end{tabular}

\begin{tabular}{|c|c|c|c|c|c|c|c|}
\hline \multirow{2}{*}{ Setor } & \multicolumn{2}{|c|}{ Bom } & \multicolumn{2}{|c|}{ Regular } & \multicolumn{2}{|c|}{ Ruim } & Total \\
\hline & $\mathrm{n}$ & $\%$ & $n$ & $\%$ & $\bar{n}$ & $\%$ & $\begin{array}{ll}\mathbf{n} & \%\end{array}$ \\
\hline Marcação de consultas & 154 & 94 & 6 & 4 & 3 & 2 & 163100 \\
\hline Recepção & 159 & 98 & 3 & 2 & 1 & 1 & 163100 \\
\hline Oftalmologistas & 163 & 100 & - & - & - & - & 163100 \\
\hline Marcação de cirurgias & 163 & 100 & - & - & - & - & 163100 \\
\hline Enfermagem & 157 & 96 & 6 & 4 & - & - & 163100 \\
\hline
\end{tabular}


A distribuição das freqüências das complicações referidas pelos pacientes durante a cirurgia na Instituição está na tabela 5 .

A maioria dos pacientes (78\%) foi submetida a facectomia pela técnica de facoemulsificação e $22 \%$ pela extração extracapsular programada, sendo que $18 \%$ apresentaram alguma complicação pós-operatória tardia, relacionada à cirurgia tabela 6.

O seguimento pós-operatório variou de 5 a 2.880 dias $(7,9$ anos), média de 658 dias ( 1,8 anos) e erro padrão da média de 24 dias.

As frequiências das acuidades visuais no pré e pós-operatórios dos olhos direito e esquerdo estão expostas nas tabelas 7 e 8 .

As acuidades visuais dos olhos foram respectivamente:

\begin{tabular}{|c|c|c|c|c|c|c|c|}
\hline \multirow{2}{*}{ Setor } & \multicolumn{2}{|c|}{ Bom } & \multicolumn{2}{|c|}{ Regular } & \multicolumn{2}{|c|}{ Ruim } & Total \\
\hline & $\mathbf{n}$ & $\%$ & $n$ & $\%$ & & & $\begin{array}{ll}\mathrm{n} & \%\end{array}$ \\
\hline Consulta & 154 & 94 & - & - & 8 & 5 & 163100 \\
\hline Cirurgia & 159 & 98 & 11 & 7 & - & - & 163100 \\
\hline Exame pós-operatório & 163 & 100 & - & - & - & - & 163100 \\
\hline
\end{tabular}

\begin{tabular}{|c|c|c|}
\hline Complicações referidas & $\mathbf{n}$ & $\%$ \\
\hline Sim - moderada & 56 & 34 \\
\hline Sim - leve & 7 & 4 \\
\hline Não & 100 & 61 \\
\hline
\end{tabular}

\begin{tabular}{|lcr|}
\hline \multicolumn{3}{|c|}{$\begin{array}{l}\text { Tabela 6. Freqüências das complicações pós-operatórias tardias, } \\
\text { relacionadas à cirurgia da catarata, dos pacientes, por olho }\end{array}$} \\
Complicações & $\mathbf{n}$ & $\%$ \\
Opacificação da cápsula posterior & 11 & 38 \\
Corectopia & 6 & 21 \\
Edema de córnea & 5 & 17 \\
Lente intra-ocular descentrada & 3 & 10 \\
Ceratite & 2 & 7 \\
Trauma Descemet & 2 & 7 \\
Total & 29 & 100 \\
\hline
\end{tabular}

\begin{tabular}{|c|c|c|c|c|}
\hline \multirow[b]{2}{*}{ Acuidade visual } & \multicolumn{2}{|c|}{ Pré-operatório } & \multicolumn{2}{|c|}{ Pós-operatório } \\
\hline & $n$ & $\%$ & $n$ & $\%$ \\
\hline I. Melhor/ igual 0,3 & 14 & 12,8 & 88 & 80,8 \\
\hline II. Entre 0,25 a 0,12 & 45 & 41,3 & 15 & 13,8 \\
\hline III. Entre 0,10 a 0,005 & 23 & 21,1 & 3 & 2,7 \\
\hline IV. Inferior a 0,05 & 27 & 24,8 & 3 & 2,7 \\
\hline Total & 109 & 100,0 & 109 & 100,0 \\
\hline$X^{2}=103,27 ; p<0,0001$ & & & & \\
\hline
\end{tabular}

\begin{tabular}{|c|c|c|c|c|}
\hline \multirow[b]{2}{*}{ Acuidade visual } & \multicolumn{2}{|c|}{ Pré-operatório } & \multicolumn{2}{|c|}{ Pós-operatório } \\
\hline & $n$ & $\%$ & $\mathbf{n}$ & $\%$ \\
\hline I. Melhor/ igual 0,3 & 9 & 12,3 & 60 & 82,2 \\
\hline II. Entre 0,25 a 0,12 & 29 & 39,7 & 9 & 12,3 \\
\hline III. Entre 0,10 a 0,005 & 19 & 26,0 & 3 & 4,1 \\
\hline IV. Inferior a 0,05 & 16 & 22,0 & 1 & 1,4 \\
\hline Total & 73 & 100,0 & 73 & 100,0 \\
\hline
\end{tabular}

Olho direito - Pré-operatório $\overline{\mathrm{X}} \pm \mathrm{DP}-0,193 \pm 0,135$ - Pósoperatório $=0,650 \pm 0,293$ "t" $=13,469-\mathrm{p}<0,0001$; e Olho Esquerdo - Pré-operatório $=\overline{\mathrm{X}} \pm \mathrm{DP}-0,217 \pm 0,197$ - Pósoperatório $=0,666 \pm 0,301$ " $\mathrm{t}$ " $=11,300-\mathrm{p}<0,0001$.

\section{DISCUSSÃO}

No Brasil, entre 1940 a 1990, a expectativa de vida aumentou de 41,5 para 67,7 anos $^{(15)}$, influindo na maior necessidade de cirurgia de catarata ${ }^{(2)}$. A maior sobrevida exige melhor função visual proporcionada aos que têm acesso à cirurgia da catarata ${ }^{(6)}$.

A dificuldade da erradicação da catarata na população de baixa renda está associada ao difícil acesso ao atendimento oftalmológico. Sugere-se a oferta pública de equipe oftalmológica com os requisitos técnicos para exames e cirurgias. Essa ação traz benefícios às regiões menos desenvolvidas ${ }^{(16)}$. Nas áreas rurais da Índia, as cirurgias de catarata são realizadas nas estruturas governamentais e em campanhas ${ }^{(3)}$. A FAV utiliza nos projetos de prevenção de cegueira e reabilitação visual uma unidade móvel, descentralizando as ações.

Tem sido observado que o grau de satisfação do usuário está associado à expectativa pré-operatória e qualidade do serviço prestado ${ }^{(7,17-18)}$. Assim, é lógico aceitar a idéia de que a indicação da Instituição, para repetição do procedimento em si, ou a indicação para parentes e amigos, constituam-se em parâmetros válidos de aferição do grau de satisfação do usuário.

Face ao fato do presente Projeto ser desenvolvido nas comunidades, e o pós-operatório ser realizado ou nas localidades atendidas ou na sede da Instituição, realizou-se a aferição da acuidade visual através da tabela de Snellen, não se incluindo as escalas de visão subnormal. Dessa forma, não foram incluídas as acuidades visuais próximas à cegueira. Esses pacientes foram categorizados como baixa visual profunda.

$\mathrm{Na}$ organização de um projeto de prevenção da cegueira e reabilitação visual deve-se considerar as características sociodemográficas da população alvo, sendo fundamental o grau de satisfação do paciente quanto à qualidade do serviço prestado e ao benefício visual obtido ${ }^{(17-20)}$. Esse perfil sofre variações com o grau de desenvolvimento do país ${ }^{(1-4,8,16,20)}$.

De forma similar à Índia ${ }^{(3)}$ e no Brasil, - em São Paulo ${ }^{(2)} \mathrm{o}$ perfil sociodemográfico caracteriza-se por paciente em idade 
superior a 60 anos, feminino e de baixo poder aquisitivo ${ }^{(2,4,8)}$. Esses dados são consistentes com os observados na casuística estudada.

Deve-se assinalar que existe, no Brasil, mais mulheres com idade superior a 40 anos (24.444.017), quando comparado aos homens $(21.867 .563)^{(15)}$. Essa maior freqüência também é evidenciada em portadoras de catarata em São Paulo $(61,5 \%)^{(8)}$, similar aos dados do presente estudo, e na Índia ${ }^{(3)}$. É possível que estando as mulheres mais envolvidas em atividades que exigem melhor coordenação visual e motora como costura e ensino aos filhos possa explicar essa maior freqüência. Além do mais, o caráter perfeccionista, mais acentuado nesse gênero, pode contribuir para uma busca mais ativa de uma melhor qualidade visual. Vale salientar a importância desse dado, visto que, sugere-se que a catarata está associada, em pacientes não diabéticos, a uma maior taxa de mortalidade, em mulheres $^{(21)}$. Mas, evidenciando a diversidade de característica demográfica, em Hong Kong, em outro estudo, observou-se o gênero masculino como o mais freqüente ${ }^{(1)}$.

Os dados relativos a escolaridade e atividades laborais detectados nesse estudo foram similares aos encontrados em São Paulo ${ }^{(2,4,8)}$, onde a população assistida apresenta características similares.

O controle de qualidade de um serviço pode ser externo, quando exercido pelos Órgãos Públicos da Saúde ${ }^{(12)}$; ou interno. Deve-se, em ambos, realizar a avaliação periódica da Instituição, como no presente estudo. Um elevado percentual de pacientes (97\%) achou a cirurgia válida, elevando a credibilidade do serviço, com retorno positivo na indicação do mesmo para parentes ou amigos.

Constatou-se, além da facilidade de acesso ao serviço especializado, pontualidade e boa qualidade de atendimento em todos os setores da Instituição, com índices em torno de 95\%, tanto no pré como no pós-operatório.

O resultado visual dos pacientes operados de catarata tem uma relação direta com a técnica cirúrgica utilizada, com as complicações operatórias, com a experiência do cirurgião e o uso de correção óptica adequada no pós-operatório ${ }^{(3,11,17,19)}$.

A melhora do resultado visual dos pacientes é mais significante quando os mesmos são submetidos à técnica da facoemulsificação ${ }^{(7,11,22-23)}$, o que tem gerado uma tendência dos cirurgiões a elegerem esse procedimento, como preferencial na maioria dos casos nos Estados Unidos ${ }^{(7,23)}$, e no Brasil ${ }^{(11,22)}$. Essa tendência também foi evidente no presente estudo. Esse procedimento, apesar de ser mais elaborado, permite que as complicações sejam minimizadas quando realizadas por cirurgiões experientes ${ }^{(11)}$.

Os resultados visuais observados nesse estudo revelam uma melhora significante nos dois olhos $(\mathrm{p}<0,0001)$. Essa melhora deve-se não só pela técnica utilizada e a supervisão da cirurgia pelo staff, assim como pelo implante de lente intraocular primário em todos os pacientes. Essa conduta não é rotineira em todos os países. Por exemplo, na Índia o uso de lente intra-ocular varia de 5,8 a 63\%. Assim, o resultado visual desses pacientes situa-se abaixo dos níveis aceitáveis. Um fator adicional importante implicado na deficiência visual desses pacientes é a falta de uso de correção óptica ${ }^{(3)}$.

No estudo atual, apesar de complicações relatadas pelos pacientes na cirurgia (39\%), estas, não tiveram uma influência maior quanto ao resultado visual final. As complicações oculares relacionadas à cirurgia da catarata, detectadas no exame oftalmológico tardio, ocorreram em 18\% dos pacientes, sendo a opacificação da cápsula posterior a complicação mais freqüente. Esse resultado está em conformidade com os dez princípios para o sucesso da cirurgia de catarata pela facoemulsificação realizada por residente com supervisão ${ }^{(22)}$. Por outro lado, na Índia observa-se uma freqüência de complicações cirúrgicas maior do que a do presente estudo, contribuindo para uma deficiência visual/cegueira importante ${ }^{(3)}$. Os maus resultados decorrem da menor habilidade do cirurgião, da qualidade do material cirúrgico - sobretudo a falta de microscópio, - da técnica operatória e do deficiente acompanhamento pós-operatório.

Como esse estudo foi conduzido com pacientes alocados de forma consecutiva, de acordo com a ordem de atendimento ao seguimento pós-operatório, ocorreram algumas limitações, comuns a estudos clínicos. $1^{\circ}$. É possível que a FAV estando distante de localidades de referência para os usuários, possa ter contribuído para abandono de alguns pacientes. $2^{\circ}$. Os pacientes, de baixo poder aquisitivo e deficiente condição cultural, podem ter opiniões diversas quanto à saúde visual e atitudes em relação ao sistema de saúde ou serviços; $3^{\circ}$. Os casos operados podem não representar as habilidades e práticas quanto à técnica utilizada na comunidade médica da região.

A análise dos dados demonstra que o modelo de atendimento foi eficiente e os recursos investidos no projeto trouxeram como retorno alto grau de satisfação dos pacientes com real melhora de sua saúde visual. Esses projetos permitem a melhoria da infra-estrutura das unidades prestadoras de serviço, incrementando, também, o treinamento dos cirurgiões nas intervenções cirúrgicas da catarata com o implante da lente intra-ocular.

Em se tratando de uma Instituição com função de assistência e ensino verificou-se que o projeto possibilita a multiplicação das ações, através da capacitação de profissionais habilitados a realizar a cirurgia da catarata utilizando modernas técnicas cirúrgicas. Esses profissionais, ao retornarem aos seus estados ou países, disseminarão os conhecimentos e melhorarão seus resultados. Além do mais, a facilidade de acesso ao serviço especializado, associado à qualidade do atendimento e o benefício na recuperação visual, provavelmente gerou a credibilidade da Instituição.

\section{AGRADECIMENTOS}

Ao professor José Natal Figueiroa, pela orientação na análise estatística do estudo. 


\section{ABSTRACT}

Purposes: To identify the social and demographic patient profile, and to evaluate the visual outcome of patients enrolled in the Medical Social Cataract Project, developed by the "Altino Ventura Foundation" - Pernambuco State, Brazil. Methods: 163 patients who underwent cataract surgery and were seen at follow-up in October and November 2002. Data were obtained by a questionnaire, which also included questions about the service qualities provided by several sectors of the institution. The patients were also evaluated as regards the visual acuity and clinical outcome. Results: The patient mean age was 68.4 years. Females were more frequent (67\%). Illiteracy was present in $20 \%$ of the patients. Housewifeliness (44\%) represented the majority of the patient activities. It was found that the relative frequency of several parameters used to assess patient satisfaction with the hospital care was greater than $95 \%$. There was statistically significant improvement, of visual acuity after surgery, in both eyes. Right eye preoperative $=\overline{\mathrm{X}} \pm \mathrm{SD}-0.193 \pm 0.135$ - postoperative $=0.650 \pm$ 0.293 " $\mathrm{t}$ " $=13.469-\mathrm{p}<0.0001$. Left eye - preoperative $=\overline{\mathrm{X}} \pm \mathrm{SD}$ $-0.217 \pm 0.197$ - postoperative $=0.666 \pm 0.301$, " $t "=11.300$ $p<0.0001$. Conclusions: The degree of satisfaction in this group of patients, with high illiteracy and excluded from the work force, was very good. An association between the quality of the provided services and benefit of visual acuity improvement was observed. These results were fundamental for greater credibility of the institution.

Keywords: Cataract/pathology; Blindness; Patient satisfaction; Quality of life; Community health services

\section{REFERÊNCIAS}

1. Michon JJ, Lau J, Chan WS, Ellwein LB. Prevalence of visual impairment, blindness, and cataract surgery in the Hong Kong elderly. Br J Ophthalmol 2002;86:133-9.

2. Zacharias LC, Graziano RM, Oliveira BFT, Hatanaka M, Cresta FB, KaraJosé N. A campanha de catarata atrai pacientes da clínica privada? Arq Bras Oftalmol 2002;65:557-61.

3. Nirmalan PK, Thulasiraj RD, Maneksha V, Rahmathullah R, Ramakrishnan R, Padmavathi A, et al. A population based eye survey of older adults in Tirunelveli district of South India: blindness, cataract surgery, and visual outcomes. Br J Ophthalmol 2002;86:505-12.
4. Kara-José N, Delgado AMN, Arieta CEL. Exeqüibilidade da cirurgia de catarata em hospital-escola: em busca de um modelo econômico. Rev Assoc Med Bras 1994;40:186-8.

5. Kara-José N, Temporini ER. Cirurgia de catarata: o porquê dos excluídos. Rev Panam Salud Pública 1999:6:242-8.

6. Rehder JRCL, Ribeiro LGIR, Mello Filho PAA, Aoki RH. Projeto Catarata: uma solução para saúde pública ocular. Rev Bras Oftalmol 1999;58:271-5.

7. Javitt JC, Steinert RF. Cataract extraction with multifocal intraocular lens implantation: a multinational clinical trial evaluating clinical, functional, and quality-of-life outcomes. Ophthalmology 2000;107:2040-8.

8. Kara-José Junior N, Schellini SA, Silva MRBM, Bruni LF, Almeida AGC. Projeto Catarata: qual a sua importância para a comunidade? Arq Bras Oftalmol 1996;59:490-3.

9. Kara-José Júnior N, Temporini ER, Kara-José N. Cataract surgery: expectations of patients assisted during a community project in São Paulo, state of São Paulo, Brazil. Rev Hosp Clin Fac Med Univ São Paulo 2001;56:163-8.

10. Busbee BG, Brown MM, Brown GC, Sharma S. Incremental cost-effectiveness of initial cataract surgery. Ophthalmology 2002;109:606-13.

11. Araújo MEXS, Chou AC, Silva CR, Oliveira LB, Neustein I. Facoemulsificação: resultados e complicações os primeiros 100 olhos. Arq Bras Oftalmol 2000;63:29-31.

12. Brasil. Ministério da Saúde. Secretaria de Assistência à Saúde. Departamento de controle e Avaliação de Sistemas. Programa Nacional de Avaliação dos Serviços Hospitalares. Resultado do Programa Nacional de Avaliação de Serviços Hospitalares 2001. [citado 2002 set 11]. Disponível em: URL: http://www.saude.gov.br.

13. Ellwein LB, Kupfer C. Aspectos estrategicos de la prevencion de la ceguera por cataratas em paises em desarrollo. Bol Oficina Sanit Panam 1996;120:491-503.

14. CDC. National Center for Health Statistics. Classifications of diseases and functioning \& disability. ICD-9-CM Rich text files (.rtf) via FTP: 2002. Dtab03.ZIP. Classification of diseases and injuries. ICD-9-CM Tabular list of diseases (FY03). 369.9 Unspecified visual loss. p.215. [citado 2003 maio 9]. Disponível em: URL: http://www.cdc.gov/nchs/icol9.htm\#RTF

15. IBGE. População. Censo demográfico 2000. Resultados preliminares da amostra. [citado 2002 nov 11]. Disponível em: URL: http://www.ibge.gov.br.

16. Oréfice F, Carvalho RC, Vieira GM, Mendes AG, França VP, Campos WR, et al. Projeto Araguaia "Viver Vendo". Rev Bras Oftalmol 1995;54:498-503.

17. Nijkamp MD, Nuijts RM, Borne B, Webers CA, van der Horst F, Hendrikse F. Determinants of patient satisfaction after cataract surgery in 3 settings. J Cataract Refract Surg 2000;26:1379-88.

18. Aldana J M, Piechulek H, Al-Sabir A. Client satisfaction and quality of health care in rural Bangladesh. [citado 2003 maio 12]. Disponível em: URL: http://www.jceh.co.uk/journal/42_9asp.

19. Khadem M. Outcomes of cataract surgery: implications for the developing world. J Med Syst 1999;23:281-9.

20. Brown MM, Brown GC, Sharma S, Busbee B, Brown H. Quality of life associated with unilateral and bilateral good vision. Ophthalmology 2001; 108:643-8

21. Reidy A, Minassian DC, Desai P, Vafidis G, Joseph J, Farrow S, Connolly A. Increased mortality in women with cataract: a population based follow up of the North London Eye Study. Br J Ophthalmol 2002;86:424-8.

22. Ghanem VC, Mannis MJ. O professor e o estudante na facoemulsificação: os dez princípios para o sucesso. Arq Bras Oftalmol 2003;66:93-9.

23. Leaming DV. Practice styles and preferences of ASCRS members - 1996 survey. J Cataract Refract Surg 1997;23:527-35.

Nos artigos enviados para publicação, o nome dos autores e suas afiliações devem estar completos. Isso facilitará a indexação e os links com as bases de dados e o CV Lates. 